may also influence the effect of benzodiazepines on normal individuals. Using groups of three volunteer men they showed a rise in "hostile" behaviour in those taking chlordiazepoxide compared with those taking a placebo. Hostility was defined as "an act, physical, verbal, ideational, whose aim is to cause an injury to or devaluation of another person." The investigators found, however, that hostility became overt only when an element of frustration was introduced into the situation, but they concluded that, while a frustrating stimulus was the activating factor, increase in chlordiazepoxide-induced hostility was a regular rather than a paradoxical effect.

As this was theoretical hostility demonstrated by psychological test procedures the application of the results to the everyday clinical use of these drugs is unclear; in practice hostile behaviour due apparently to benzodiazepines is something which most clinicians might choose to regard as being so rare as to be of little significance. However, the possibility of such drugs having a disinhibiting effect may have to be borne in mind, for in those patients who are subject to environmental frustration use of the drug may lead to aggressive or hostile behaviour. Such an event was first reported in 1960 by Ingram and Timbury ${ }^{8}$ in a patient taking chlordiazepoxide, who physically assaulted his wife for the first time in their 20 years of marriage. Again, patients undergoing chronic renal haemodialysis who are given diazepam to calm their apprehension often react more adversely to the frustrating nature of the procedure than they might otherwise be expected to do. $^{9}$ If these are valid conclusions then they provide clear evidence that there may be dangers in the growing practice of prescribing minor tranquillisers for anxiety and tension brought about by environmental frustrations and disturbed interpersonal relationships.

\footnotetext{
1 Fox, K. A., and Snyder, R. L., Fournal of Comparative and Physiological Psychology, 1969, 69, 663.

2 Guatini, A. G., Marucci, F., and Garattini, S., Psychopharmacologia, $1971,19,241$.

3 Chance, M. R. A., Fournal of Pharmacology, 1946, 87, 214.

4 Tobin, J. M., and Lewis, N. D. C., Fournal of the American Medical Association, 1960, 174, 1242.

5 Hollister, L. E., Motzenbecker, F. P., and Degan, R. O., Psychopharmacologia, 1961, 2, 63.

6 Feldman, P. E., fournal of Neuropsychiatry, 1962, 3, S.62.

7 Salzman, C., et al., Archives of General Psychiatry, 1974, 31, 401.

8 Ingram, I. M., and Timbury, G. D., Lancet, 1960, 2, 766.

9 Morgan, D. H., Department of Psychiatry, Birmingham University, unpublished observation, 1974 .
}

\section{Vision and Driving}

Driving a car is a complex skill, calling on a wide variety of perceptions and predictions. Generally these are based on experience acquired from both driving and other situations such as the judgement of speed and distance made while walking. However, since the car removes proprioceptive information of movement relative to the ground vision is all-important.

Visual judgement of distance depends on direct signals and deductions. The direct signals include the convergence angle of fixation of the two eyes, which gives the distance of only one object at a time; stereoscopic fusion of the disparate retinal images, which gives depth across a wide field by neural cross-correlation of corresponding retinal points; and accommodation of the lens-a weak cue. These are important only for rather short distances and are not normally vital for driving. Single-eyed drivers are probably handicapped only when manoeuvring and judging final stopping distances, and then not seriously.

The indirect information comes from experience of the perspective convergence with distance, especially of parallel lines such as the edges of roads; and the perspective shrinking of texture from the road surface with distance. Lack of intervening textured surfaces impairs distance perception dramatically at night and in fog. Additional clues may come from loss of fine expected detail due to the eye's limited resolution (but this may be affected by changes of road surface and by uneven lighting, and so is not reliable), and the size of familiar objects such as cars or the spacing of rear lights. Bright lights appear nearer than dim lights, and this can be misleading; and light colours may appear slightly nearer than dark colours. Red appears somewhat nearer than blue.

Relative motion gives many more cues, but these also depend on knowledge of or assumptions about surrounding objects. Motion parallax is the relative shift of nearer against further objects. It is complicated by changes in the fixation distance of the eyes-the world rotates around the point of fixation during observer movement. In fog or poor lighting, ${ }^{1}$ when distant objects are not visible, this important information may be lost. Expansion of the retinal image is most important for estimating closing rates. In walking, absolute distance can also be estimated from the incremental increase in the size of the retinal image with each step taken; but this computation may not be reliable for driving, when proprioceptive information of movement from the ground is lost. Further evidence comes from the changes in shape of retinal images, especially of objects having straight lines and definite corners. The ability of the visual system to maintain the fixed shape of objects during rotation, as when passing cars or houses by the road, is remarkable and by no means fully understood.

Judgement of speed is also based on direct and indirect signals. The most obvious is the velocity of shift of the image across the retina-which includes the increase in size of the retinal image with decreasing closing distance. A second factor is movement of the eyes as they follow moving objects. Though eye movements sweep the image across the retinae the world does not swing round with voluntary eye movements, as the retinal and eye movement signals are neurally cancelled -though not in nystagmus, when the visual world does become unstable and orientation can be lost.

As for distance, knowledge of or assumptions about objects can affect perceived motion. Motion parallax is important for detecting relative movement and is related both to actual and to perceived distances. The regular spacing of cats' eyes and telegraph poles can indicate speed and is especially useful in fog. The recent scheme of lines painted across the road at decreasing intervals before some junctions makes the driver slow down as he assumes them to have roughly equal spacing. This is a clever use of an illusion to control behaviour.

The importance for safe driving of knowledge of the sizes and shapes of objects and distributions of texture can, then, hardly be overstressed, but they must be discovered by experiment. Equally important is perceptual prediction, and again experience is the best teacher. In normal driving conditions decisions are based not so much on visual signals as on expectations of what is about to happen. In many skilled activities prediction can reduce-often to zero-the neural reaction-time of around 0.5 seconds; and it allows the skill to be maintained with intermittent sensory inputs-so the eyes can be free to search for relevant data and to rest from staring continuously at the road ahead. Since predictions cannot be infallible-and for driving they include other driver's predictions and they may be wrong-some accidents must be expected in unusual situations.

1 Weale, R. A., British Medical fournal 1974, 4, 149. 